\title{
Nonelliptic Approximation of a Class of Partial Differential Equations with Neumann Boundary Condition
}

\author{
By V. Girault
}

\begin{abstract}
This paper is devoted to the numerical resolution of a class of linear partial differential equations with an inhomogeneous Neumann boundary condition. A first order quadrilateral finite element method is used, together with a one-point integration formula. The resulting scheme is simple and widely used but its theory is not classical, in a sense described as "nonelliptic". An important boundary value theorem is derived, in order to handle the Neumann condition. An error bound shows that the scheme is of order one.
\end{abstract}

1. Introduction. Let $\Omega$ be a bounded domain in the $(x, y)$-plane with sufficiently smooth boundary $\Gamma$. The purpose of this paper is the approximation of a general elliptic equation in $\Omega$ with an inhomogeneous Neumann boundary condition:

Continuous Neumann's Problem. Find a function $u$ satisfying

$$
\begin{array}{r}
-\frac{\partial}{\partial x}\left(a_{11} \frac{\partial u}{\partial x}+a_{12} \frac{\partial u}{\partial y}\right)-\frac{\partial}{\partial y}\left(a_{21} \frac{\partial u}{\partial x}+a_{22} \frac{\partial u}{\partial y}\right) \\
+b_{1} \frac{\partial u}{\partial x}+b_{2} \frac{\partial u}{\partial y}+d u=f \text { in } \Omega
\end{array}
$$

with the boundary condition

$$
\left(a_{11} \frac{\partial u}{\partial x}+a_{12} \frac{\partial u}{\partial y}\right) \cos (\vec{n}, x)+\left(a_{21} \frac{\partial u}{\partial x}+a_{22} \frac{\partial u}{\partial y}\right) \cos (\vec{n}, y)=g \quad \text { on } \Gamma,
$$

where

$$
\begin{aligned}
& a_{i j}, b_{i} \text { and } d \text { are given functions in } L^{\infty}(\Omega), f \text { is } \\
& \text { given in } L^{2}(\Omega) \text { and } g \text { is given in } L^{2}(\Gamma),
\end{aligned}
$$

and where $\cos (\vec{n}, x)$ and $\cos (\vec{n}, y)$ denote the direction cosines of the exterior normal to $\Gamma$.

We propose to approximate this problem by an isoparametric quadrilateral finite element method of order one, coupled with a one-point integration formula. This method gives rise to a very simple scheme which, originally, was derived by several authors as a generalization of finite differences on irregular networks. Since then, it

Received June 4, 1974; revised February 13, 1975. 35 A15.

AMS (MOS) subject classifications (1970). Primary 65N30, 65N05, 65N15; Secondary

Key words and phrases. Finite elements, discrete Green's formula, boundary values. 
has been widely and successfully applied in practice to a variety of problems (cf. for instance, Hirt, Amsden \& Cook [5]). In a previous work, we have studied its application to a Dirichlet boundary value problem (cf. Girault [4]).

The main originality of our method is that it violates Strang's ellipticity condition (cf. Strang \& Fix [10]), and for this reason, we shall call it nonelliptic. Because of the nonellipticity, this method cannot be studied by the now classical theory of finite element methods and numerical integration (cf. Ciarlet \& Raviart [3]). Therefore, its theory must be carried out through an entirely different approach. In order to explain why Strang's condition does not hold, let us recall very briefly the main steps of isoparametric finite element methods.

$\left(1^{\circ}\right)$. The continuous problem is put into an equivalent variational form: Find a function $u$ in $V$ satisfying

$$
a(u, v)=L(v) \quad \forall v \in V,
$$

for a suitable space $V$ and form $L$, and a suitable positive definite form $a$.

$\left(2^{\circ}\right)$. The domain $\Omega$ is subdivided into finite elements which in our case are quadrilaterals; and the variational problem is formulated in terms of a finite-dimensional subspace $V_{h}$ of $V$, consisting of simple functions defined on each finite element. Thus, problem (1.4) is approximated by: Find a function $\bar{u}_{h}$ in $V_{h}$ satisfying

$$
a\left(\bar{u}_{h}, v_{h}\right)=L\left(v_{h}\right) \quad \forall v_{h} \in V_{h} .
$$

It is now well known that isoparametric finite element methods are very well adapted to the resolution of second order boundary value problems on domains with curved boundaries. However, such methods require the evaluation of a great many integrals which are often difficult, if not impossible, to calculate exactly. As an illustration, in problem (1.1), (1.2), quadrilateral finite elements involve the integration of rational functions over rectangles, and the Neumann boundary condition leads to line integrals taken over curves which practically never coincide with sides of quadrilaterals. None of these integrals can be calculated exactly. Therefore, for all practical purposes, finite element methods are nearly always coupled with one or more adequate integration formulas. Hence, a third step is added to the preceding two.

$\left(3^{\circ}\right)$. The integrals occurring in (1.5) are computed with suitable integration formulas, and (1.5) is approximated by: Find a function $u_{h}$ in $V_{h}$ such that

$$
a_{h}\left(u_{h}, v_{h}\right)=L_{h}\left(v_{h}\right) \quad \forall v_{h} \in V_{h} .
$$

Now, if the quadrature formula uses enough integration points, then $a_{h}\left(v_{h}, v_{h}\right)$ is uniformly equivalent to $a\left(v_{h}, v_{h}\right)$ on $V_{h}$ and Strang's ellipticity condition is satisfied. Otherwise, if the quadrature formula, however precise, has too few points then this equivalence is practically never uniform; and, therefore, scheme (1.6) is nonelliptic.

Our approximation falls into the last category because a one-point integration formula does not have enough points to make the resulting scheme elliptic. As mentioned above, this is sufficient to break down the existing theory of finite element approximation. The major effort of this paper is to show that, nevertheless, the error in our method is of the order of $h$. A new theory is introduced, but the variational 
framework is maintained. Because of the Neumann condition, this requires, in particular, a careful analysis of the approximation near the boundary.

The next section states precisely what is meant by nonelliptic approximation in general and sets problem (1.1), (1.2) in a variational frame. Sections 3 and 4 are devoted to the approximation of the resulting problem. Section 5 gives an important discrete boundary value theorem! The results of these sections are used, in Section 6, to derive an error estimate for our approximation.

The presentation of this paper differs from that of our previous one (cf. Girault [4]) in that we follow here more closely the finite element formalism. Although the two papers are independent, we have tried, as much as possible, to use the same notation.

\section{Theoretical Framework.}

2.1. General Nonelliptic Approximation. Let $V$ be a real Hilbert space, with norm denoted by $\|\cdot\|, L$ a member of the dual space $V^{\prime}$ of $V$, and $a(u, v)$ a bilinear, continuous and elliptic form on $V \times V$, that is,

(i) there exists a constant $P$ such that

$$
|a(u, v)| \leqslant P\|u\|\|v\| \quad \forall u, v \in V,
$$

(ii) there exists a constant $\alpha>0$ such that

$$
a(v, v) \geqslant \alpha\|v\|^{2} \quad \forall v \in V .
$$

We propose to approximate the following problem:

Abstract Continuous Problem. Find $u$ in $V$ satisfying

$$
a(u, v)=L(v) \quad \forall v \in V .
$$

Thanks to the above hypotheses, this problem has a unique solution $u$ (cf. Lions [6]). We assume that $u$ belongs to some subspace $V$ of $V$; this amounts in fact to making a regularity hypothesis on $u$.

Let $h$ be a positive parameter intended to tend to zero, and for each $h$, let $V_{h}$ be a finite-dimensional subspace of $V$. Let $v_{h} \mapsto\left\|v_{h}\right\|_{h}$ be a mapping defined on $V+V_{h}$ such that $\|\cdot\|_{h}$ is a norm on $V_{h}$. Let $a_{h}\left(u_{h}, v_{h}\right)$ be a bilinear form defined on $\left(V+V_{h}\right)$ $\times\left(V+V_{h}\right)$ satisfying conditions similar to (i) and (ii):

(2.2) There exists a constant $M$, independent of $h$, such that

$$
\left|a_{h}\left(u_{h}, v_{h}\right)\right| \leqslant M\left\|u_{h}\right\|_{h}\left\|v_{h}\right\|_{h} \quad \forall u_{h} \in V+V_{h} \text { and } \forall v_{h} \in V_{h} .
$$

(2.3) There exists a constant $\beta>0$, independent of $h$, such that

$$
a_{h}\left(v_{h}, v_{h}\right) \geqslant \beta\left\|v_{h}\right\|_{h}^{2} \quad \forall v_{h} \in V_{h} .
$$

Finally, let $L_{h}$ be a linear form on $V+V_{h}$ satisfying the following condition:

(2.4) There exists a constant $\mu$, independent of $h$, such that

$$
\left|L_{h}\left(v_{h}\right)\right| \leqslant \mu\left\|v_{h}\right\|_{h} \quad \forall v_{h} \in V_{h} .
$$

We approximate our continuous problem as follows:

Abstract Approximate Problem. Find $u_{h}$ in $V_{h}$ such that

$$
a_{h}\left(u_{h}, v_{h}\right)=L_{h}\left(v_{h}\right) \quad \forall v_{h} \in V_{h} \text {. }
$$

Definition 2.1. We say that the above approximation is nonelliptic if form $a_{h}$ is 
not uniformly elliptic (with respect to $h$ ) on $V_{h}$ for the norm $\|\cdot\|$ of $V$.

From now on, we shall assume that approximation (2.5) is nonelliptic. Then, we cannot apply the general theory of internal approximation (cf. Ciarlet \& Raviart [3]); but we have the following theorem, which is an easy extension of a result due to Strang (cf. Strang [9]):

THEOREM 2.1. Let hypotheses (2.2) and (2.3) be satisfied. Then problem (2.5) has a unique solution $u_{h}$, and we have the error estimate:

$$
\left\|u-u_{h}\right\|_{h} \leqslant C\left\{\inf _{v_{h} \in V_{h}}\left\|u-v_{h}\right\|_{h}+\sup _{w_{h} \in V_{h} ; w_{h} \neq 0} \frac{1}{\left\|w_{h}\right\|_{h}}\left|L_{h}\left(w_{h}\right)-a_{h}\left(u, w_{h}\right)\right|\right\},
$$

where $u$ is the solution of problem (2.1) and $C$ is a constant depending only on $M$ and $\beta$.

Proof. As in the case of the continuous problem (2.1), the existence and uniqueness of $u_{h}$ are a consequence of the continuity and ellipticity of $a_{h}$ and the continuity of $L_{h}$ (cf. Lions [6]), but none of these properties need to be uniform with respect to $h$.

Let $v_{h}$ belong to $V_{h}$. Hypothesis (2.3) implies that

$$
\left\|u_{h}-v_{h}\right\|_{h} \leqslant \frac{1}{\beta} \sup _{w_{h} \in V_{h} ; w_{h} \neq 0} \frac{1}{\left\|w_{h}\right\|_{h}}\left|a_{h}\left(u_{h}-v_{h}, w_{h}\right)\right| .
$$

Now

$$
\begin{aligned}
a_{h}\left(u_{h}-v_{h}, w_{h}\right) & =a_{h}\left(u_{h}, w_{h}\right)-a_{h}\left(v_{h}, w_{h}\right) \\
& =L_{h}\left(w_{h}\right)-a_{h}\left(u, w_{h}\right)+a_{h}\left(u, w_{h}\right)-a_{h}\left(v_{h}, w_{h}\right) .
\end{aligned}
$$

Therefore, $\left|a_{h}\left(u_{h}-v_{h}, w_{h}\right)\right| \leqslant\left|L_{h}\left(w_{h}\right)-a_{h}\left(u, w_{h}\right)\right|+\left|a_{h}\left(u-v_{h}, w_{h}\right)\right|$, and thanks to (2.2), we have:

$$
\left\|u_{h}-v_{h}\right\|_{h} \leqslant \frac{1}{\beta} \sup _{w_{h} \in V_{h} ; w_{h} \neq 0} \frac{1}{\left\|w_{h}\right\|_{h}}\left\{\left|L_{h}\left(w_{h}\right)-a_{h}\left(u, w_{h}\right)\right|+M\left\|u-v_{h}\right\|_{h}\left\|w_{h}\right\|_{h}\right\} .
$$

Formula (2.6) follows easily from this inequality.

Remarks 2.1. ( $\left.1^{\circ}\right)$ The expression $\inf _{v_{h} \in V_{h}}\left\|u-v_{h}\right\|_{h}$ represents the error committed on projecting function $u$ of $V$ on $V_{h}$. The second expression, $\sup _{w_{h} \in V_{h}}\left(1 /\left\|w_{h}\right\|_{h}\right)\left|L_{h}\left(w_{h}\right)-a_{h}\left(u, w_{h}\right)\right|$, gives a measure of the consistency of (2.5) with the exact problem (2.1).

$\left(2^{\circ}\right)$ Although property (2.4) is not one of the hypotheses of Theorem 2.1 , it is included here since it is obviously necessary to the consistency of (2.5) with (2.1).

2.2. Variational Formulation. In order to apply the preceding approximation to problem (1.1), (1.2), we must first write it in a weakly equivalent variational form (cf. Lions [8]):

Variational Neumann Problem. Find $u$ in $H^{1}(\Omega)$ satisfying

$$
a(u, v)=L(v) \quad \forall v \in H^{1}(\Omega),
$$

where 


$$
\begin{gathered}
a(u, v)=\int_{\Omega}\left\{\left(a_{11} \frac{\partial u}{\partial x}+a_{12} \frac{\partial u}{\partial y}\right) \frac{\partial v}{\partial x}\right. \\
+\left(a_{21} \frac{\partial u}{\partial x}+a_{22} \frac{\partial u}{\partial y}\right) \frac{\partial v}{\partial y} \\
\left.+\left(b_{1} \frac{\partial u}{\partial x}+b_{2} \frac{\partial u}{\partial y}+d u\right) v\right\} d x d y, \\
L(v)=\int_{\Omega} f v d x d y+\int_{\Gamma} g v d \Gamma
\end{gathered}
$$

and

$$
a_{i j}, b_{i} \text { and } d \in L^{\infty}(\Omega), f \in L^{2}(\Omega) \text { and } g \in L^{2}(\Gamma) .
$$

As usual, $H^{m}(\Omega)$ denotes the classical Sobolev space of functions $v$ such that $v$ and all its derivatives of order less than or equal to $m$ belong to $L^{2}(\Omega)$. For example, $H^{1}(\Omega)$ is a Hilbert space for the norm

$$
\|v\|_{H^{1}(\Omega)}=\left\{\|v\|_{L^{2}(\Omega)}^{2}+\left\|\frac{\partial v}{\partial x}\right\|_{L^{2}(\Omega)}^{2}+\left\|\frac{\partial v}{\partial y}\right\|_{L^{2}(\Omega)}^{2}\right\}^{1 / 2} .
$$

Let us make the following assumption on the coefficients:

Hypothesis $\mathrm{H} 1$ (ellipticity). There exists a constant $\gamma>0$ such that

$$
\left.\begin{array}{l}
\sum_{i, j=1}^{2} a_{i j}(x, y) \xi_{i} \xi_{j} \geqslant \gamma \sum_{i=1}^{2} \xi_{i}^{2} \quad \forall\left(\xi_{1}, \xi_{2}\right) \in R^{2} \text { and } \\
d(x, y) \geqslant \gamma
\end{array}\right\} \text { a.e. in } \Omega .
$$

With the above assumptions, it can be shown (cf. Lions [7]) that form $L$ defined by (2.8) belongs to the dual space of $H^{1}(\Omega)$; if, moreover, $\left\|b_{i}\right\|_{L^{\infty}(\Omega)}, i=1,2$, are small enough, then form $a$ defined by (2.7) satisfies (i) and (ii). This can be summarized as follows:

Proposition 2.1. If $\Gamma$ is sufficiently smooth, $a_{i j}, b_{i}$ and $d \in L^{\infty}(\Omega), f \in L^{2}(\Omega)$, $g \in L^{2}(\Gamma)$ and Hypothesis $\mathrm{H} 1$ is satisfied, then problem (2.1) (2.7) (2.8) has a unique solution, provided $\left\|b_{i}\right\|_{L^{\infty}(\Omega)}$ are sufficiently small.

We shall take $V=H^{2}(\Omega) \cap C^{1}(\bar{\Omega})$. Finally, in order to study properly the error estimate (2.6), it will be convenient to strengthen the regularity of the coefficients and boundary. We shall add the following hypotheses:

Hypothesis $\mathrm{H} 2$ (regularity of coefficients).

$$
a_{i j} \in C^{2}(\bar{\Omega}) \quad \text { for } i, j=1,2, \quad b_{i} \in C^{1}(\bar{\Omega}) \quad \text { for } i=1,2,
$$

$d$ and $f \in \mathcal{C}^{1}(\bar{\Omega})$ and $g \in \mathcal{C}^{1}(C)$ for each arc $C$ of $\Gamma$.

Hypothesis $\mathrm{H} 3$ (regularity of boundary). $\Gamma$ consists of a finite number of simple arcs of class $C^{2}$. At the angular points of $\Gamma$, the angle between the two tangents is greater than an angle $\theta_{0}$ with $0<\theta_{0} \leqslant \pi$ (cf. Figure 1). 

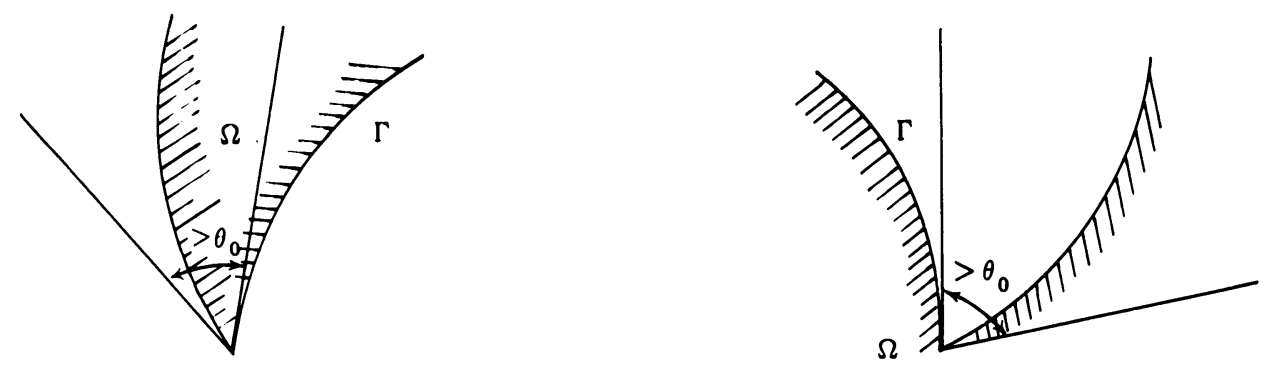

Figure 1

\section{Nonelliptic Approximation of Neumann's Problem.}

3.1. The Finite Element Method. Let $\hat{K}$ denote the unit square, with vertices $\left\{\hat{a}_{i}\right\}_{i=1}^{4}$, of the reference space $(\hat{x}, \hat{y})$, and let $K^{*}$ be any plane quadrilateral with vertices $\left\{a_{i, K}\right\}_{i=1}^{4}$ (cf. Figure 2). Let $Q_{1}$ denote the space of bilinear polynomials in $\hat{x}$ and $\hat{y}$. Then, there exists one and only one mapping $F_{K}$ in $Q_{1}^{2}$ such that $F_{K}\left(a_{i}\right)=a_{i, K}$ for $1 \leqslant i \leqslant 4$.

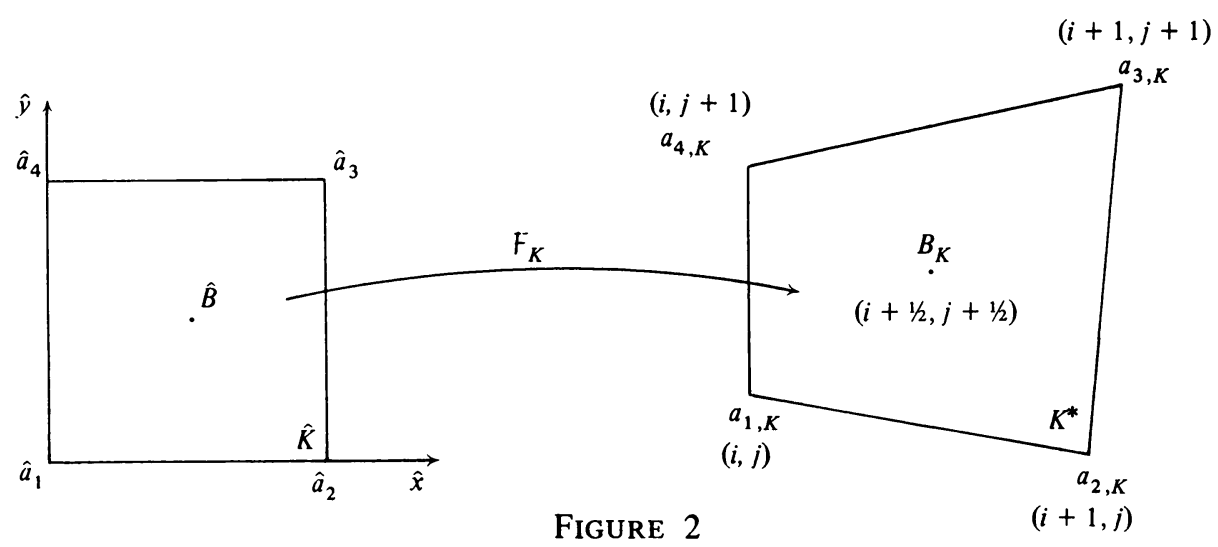

Now, let $Q_{h}$ be a quadrangulation of $\Omega$ made of quadrilaterals or cells $K^{*}$ with sides smaller than $h$. Let $\Omega_{h}^{*}=\bigcup_{K^{*} \in \Omega_{h}} \bar{K}^{*}$ and $\Gamma_{h}=\partial \Omega_{h}^{*}$. We assume that the quadrangulation is locally regular, that is (cf. Ciarlet \& Raviart [3]):

(3.1) all quadrilaterals $K^{*}$ are strictly convex;

(3.2) there exists a constant $\sigma_{0}>0$, independent of $h$, such that

$$
h_{K}^{\prime} \geqslant \sigma_{0} h \text { and } 1-\gamma_{K} \geqslant \sigma_{0} \quad \forall K^{*} \in Q_{h},
$$

where $h_{K}^{\prime}$ denotes the length of the smallest side of $K^{*}$ and $\gamma_{K}=\max \left|\cos \theta_{K}\right|$ where $\theta_{K}$ represent the angles of $K^{*}$.

Moreover, we assume that:

(3.3) $\Gamma_{h}$ is a polygonal line of chords approximating $\Gamma$, and all angular points of $\Gamma$ are vertices of $\Gamma_{h}$;

(3.4) the vertices of $2_{h}$ can be numbered by a pair of indices $(i, j)$.

Hypothesis (3.3) implies that $\Omega_{h}^{*}$ approximates $\Omega$; more precisely,

$$
\sup _{(x, y) \in \Omega_{\hbar}^{*}} \operatorname{dist}((x, y), \Omega)=O\left(h^{2}\right) .
$$


Thus, there exists a compact set $\widetilde{\Omega}$ containing both $\Omega$ and $\Omega_{h}^{*}$.

Hypothesis (3.1) implies that each $F_{K}$ is invertible and we define space $V_{h}$ as follows:

$$
V_{h}=\left\{v_{h} \in C^{0}\left(\Omega_{h}^{*}\right) \mid v_{\left.h\right|_{K}}=q \circ F_{K}^{-1} \forall q \in Q_{1}\right\}
$$

Also, let $V_{h}^{\prime}=\left\{v_{h}\left|\Gamma_{h}\right| v_{h} \in V_{h}\right\}$.

Finally, for each real function $u$ defined at the vertices of $V_{h}$, let $r_{h} u$ be the function of $V_{h}$ which takes the same value as $u$ at the vertices of $2_{h}$.

Remark 3.1. The situation here differs slightly from that of Section 2 since in general $V_{h} \not \subset H^{1}(\Omega)$. In practice, this "variational crime" is not very important because $\Omega$ and $\Omega_{h}^{*}$ are fairly alike. Theoretically, the difficulty may be overcome by replacing $H^{1}(\Omega)$ by $H^{1}(\widetilde{\Omega})$ and extending functions $a_{i j}, b_{i}, d$ and $f$ to obtain functions, still denoted by $a_{i j}, b_{i}, d$ and $f$, belonging, respectively, to $C^{2}(\widetilde{\Omega})$ and $C^{1}(\widetilde{\Omega})$. We still denote by $V$ the space $H^{2}(\widetilde{\Omega}) \cap C^{1}(\widetilde{\Omega})$, and we extend continuously all functions of $V_{h}$ to $\widetilde{\Omega}$, so that $V_{h} \subset H^{1}(\widetilde{\Omega})$.

Let us define two integration formulas on $K^{*}$ :

$$
\left.\begin{array}{l}
\int_{K^{*}} \varphi(x, y) d x d y \sim J_{K}(\hat{B}) \varphi\left(B_{K}\right)=F_{1}(\varphi) \\
\int_{K^{*}} \varphi(x, y) d x d y \sim \sum_{i=1}^{4} \frac{1}{4} J_{K}\left(\hat{a}_{i}\right) \varphi\left(a_{i, K}\right)=F_{2}(\varphi)
\end{array}\right\} \forall \varphi \in C^{0}\left(K^{*}\right),
$$

where $J_{K}$ denotes the Jacobian of $F_{K}, \hat{B}$ the mean center of $\hat{K}$ and $B_{K}$ the mean center of $K^{*}$. On $\hat{K}$, these formulas are both exact for all polynomials of $Q_{1}$. Let $K^{\prime}$ denote a side of $K^{*}$, for instance segment $\left[a_{p, K}, a_{q, K}\right]$. Let $a_{p q, K}$ stand for its midpoint and $l_{K}$, its length. Formulas $F_{1}$ and $F_{2}$ induce two formulas, $F_{1}^{\prime}$ and $F_{2}^{\prime}$, on $K^{\prime}$ :

$$
\left.\begin{array}{l}
\int_{K^{\prime}} \varphi(x, y) d \sigma \sim l_{K^{\prime}} \varphi\left(a_{p q, K}\right)=F_{1}^{\prime}(\varphi) \\
\int_{K^{\prime}} \varphi(x, y) d \sigma \sim \frac{1}{2} l_{K^{\prime}}\left\{\varphi\left(a_{p, K}\right)+\varphi\left(a_{q, K}\right)\right\}=F_{2}^{\prime}(\varphi)
\end{array}\right\} \forall \varphi \in C^{0}\left(K^{\prime}\right) .
$$

Here, $F_{1}^{\prime}$ and $F_{2}^{\prime}$ are exact for all polynomials of degree one.

Remarks 3.2. $\left(1^{\circ}\right)$ Let $S_{K}^{*}$ denote the area of $K^{*}$. Then $J_{K}(\hat{B})=S_{K}^{*}$.

$\left(2^{\circ}\right)$ Let $v_{h} \in V_{h}$, then

$$
v_{h}\left(B_{K}\right)=\frac{1}{4} \sum_{i=1}^{4} v_{h}\left(a_{i, K}\right) \quad \text { and } \quad v_{h}\left(a_{p q, K}\right)=\frac{1}{2}\left(v_{h}\left(a_{p, K}\right)+v_{h}\left(a_{q, K}\right)\right) \text {. }
$$

$\left(3^{\circ}\right)$ From (3.1) and (3.2), it follows that there exist two positive constants, $c_{0}$ and $\sigma_{0}$, independent of $h$, such that

$$
\begin{aligned}
& \inf _{(\hat{x}, \hat{y}) \in \hat{K}} J_{K}(\hat{x}, \hat{y}) \geqslant c_{0} h^{2} \forall K^{*} \in Q_{h}, \text { and } \\
& l_{K^{\prime}} \geqslant \sigma_{0} h \quad \forall K^{\prime} \in \Gamma_{h} .
\end{aligned}
$$


Moreover, it is obvious that there exists a constant $C_{0}$, independent of $h$, such that

$$
\sup _{(\hat{x}, \hat{y}) \in \hat{K}} J_{K}(\hat{x}, \hat{y}) \leqslant C_{0} h^{2} \quad \forall K^{*} \in Q_{h} .
$$

Thus we have the important bounds

$$
\begin{array}{cc}
c_{0} h^{2} \leqslant S_{K}^{*} \leqslant C_{0} h^{2} & \forall K^{*} \in Q_{h}, \\
\sigma_{0} h \leqslant l_{K^{\prime}} \leqslant h & \forall K^{\prime} \in \Gamma_{h} .
\end{array}
$$

We approach Neumann's problem as follows:

Approximate Neumann's Problem. Find $u_{h}$ in $V_{h}$ such that

$$
a_{h}\left(u_{h}, v_{h}\right)=L_{h}\left(v_{h}\right) \quad \forall v_{h} \in V_{h},
$$

where

$$
\begin{aligned}
a_{h}\left(u_{h}, v_{h}\right)=\sum_{K^{*} \in Q_{h}}\left\{F_{1}\right. & {\left[\left(a_{11} \frac{\partial u_{h}}{\partial x}+a_{12} \frac{\partial u_{h}}{\partial y}\right) \frac{\partial v_{h}}{\partial x}\right.} \\
& +\left(a_{21} \frac{\partial u_{h}}{\partial x}+a_{22} \frac{\partial u_{h}}{\partial y}\right) \frac{\partial v_{h}}{\partial y} \\
& \left.\left.+\left(b_{1} \frac{\partial u_{h}}{\partial x}+b_{2} \frac{\partial u_{h}}{\partial y}\right) v_{h}\right]+F_{2}\left(d u_{h} v_{h}\right)\right\},
\end{aligned}
$$

$$
L_{h}\left(v_{h}\right)=\sum_{K^{*} \in Q_{h}} F_{1}\left(f v_{h}\right)+\sum_{K^{\prime} \in \Gamma_{h}} F_{1}^{\prime}\left(g v_{h}\right)
$$

or

$$
L_{h}\left(v_{h}\right)=\sum_{K^{*} \in Q_{h}} F_{2}\left(f v_{h}\right)+\sum_{K^{\prime} \in \Gamma_{h}} F_{1}^{\prime}\left(g v_{h}\right)
$$

Remarks 3.3. ( $\left.1^{\circ}\right)$ Strictly speaking, as $g$ is only defined on $\Gamma$, formula $F_{1}^{\prime}$ can only be used when $a_{p q, K}$ belongs to $\Gamma$. However, according to (3.5), there exists at least one point $\tilde{a}_{p q, K}$ on $\Gamma$ such that $\operatorname{dist}\left(\tilde{a}_{p q, K}, a_{p q, K}\right)=O\left(h^{2}\right)$. We then agree to use $g\left(\tilde{a}_{p q, K}\right)$ in $F_{1}^{\prime}$.

$\left(2^{\circ}\right)$ It will be shown in Section 6 that the use of $\left(3.9^{\prime}\right)$ instead of (3.9) does not change the error estimate.

3.2. Preliminary Analysis of the Approximation. Let us define on $V_{h}$ the scalar product

$$
\left(u_{h}, v_{h}\right)_{h}=\sum_{K^{*} \in Q_{h}} F_{2}\left(u_{h} v_{h}\right)
$$

and the norms

$$
\begin{aligned}
& \left|u_{h}\right|_{h}=\left(u_{h}, u_{h}\right)_{h}^{1 / 2} \\
& \left\|u_{h}\right\|_{h}=\left(\sum_{K^{*} \in Q_{h}} F_{1}\left\{\left(\frac{\partial u_{h}}{\partial x}\right)^{2}+\left(\frac{\partial u_{h}}{\partial y}\right)^{2}\right\}+\left|u_{h}\right|_{h}^{2}\right)^{1 / 2}
\end{aligned}
$$


Similarly, we define on $V_{h}^{\prime}$ the scalar product

$$
\left(u_{h}, v_{h}\right)_{\Gamma_{h}}=\sum_{K^{\prime} \in \Gamma_{h}} F_{2}^{\prime}\left(u_{h} v_{h}\right)
$$

and the associated norm $\left|u_{h}\right|_{\Gamma_{h}}=\left(u_{h}, u_{h}\right)_{\Gamma_{h}}^{1 / 2}$.

Thanks to the choice of the integration points in $F_{2}$ and $F_{2}^{\prime}$, we have the following lemma:

LEMmA 3.1. The mappings $u_{h} \mapsto\left|u_{h}\right|_{h}$ and $u_{h} \mapsto\left\|u_{h}\right\|_{h}$ are norms on $V_{h}$. Similarly, $u_{h} \mapsto\left|u_{h}\right|_{\Gamma_{h}}$ is a norm on $V_{h}^{\prime}$.

It can be shown by counterexample (cf. Girault [4]) that the norm defined above, $\|\cdot\|_{h}$, is not uniformly equivalent on $V_{h}$ to norm $\|\cdot\|$; hence our approximation is nonelliptic.

The following notation will be useful: let

$$
\begin{gathered}
{[\varphi, \psi]_{h}=\sum_{K^{*} \in Q_{h}} F_{1}(\varphi \psi) \quad \text { for } \varphi, \psi \text { defined on } \widetilde{\Omega},} \\
{[\varphi, \psi]_{\Gamma_{h}}=\sum_{K^{\prime} \in \Gamma_{h}} F_{1}^{\prime}(\varphi \psi) \text { for } \varphi, \psi \text { defined on } \Gamma_{h},} \\
{\left[u_{h}\right]_{h}=\left[u_{h}, u_{h}\right]_{h}^{1 / 2} \text { and }\left[u_{h}\right]_{\Gamma_{h}}=\left[u_{h}, u_{h}\right]_{\Gamma_{h}}^{1 / 2} .}
\end{gathered}
$$

LemmA 3.2. There exists a constant $C$, independent of $h$, such that

$$
\left[u_{h}\right]_{h} \leqslant C\left|u_{h}\right|_{h} \quad \forall u_{h} \in V_{h} .
$$

Moreover,

$$
\left[u_{h}\right]_{\Gamma_{h}} \leqslant\left|u_{h}\right|_{\Gamma_{h}} \quad \forall u_{h} \in V_{h}^{\prime} .
$$

Proof. For (3.10), it suffices to show that, for each $K^{*}$ in $2_{h}$,

$$
F_{1}\left(u_{h}^{2}\right) \leqslant C^{2} F_{2}\left(u_{h}^{2}\right)
$$

But

$$
\begin{aligned}
F_{1}\left(u_{h}^{2}\right) & =u_{h}^{2}\left(B_{K}\right) S_{K}^{*}=\left\{\frac{1}{4} \sum_{i=1}^{4} u_{h}\left(a_{i, K}\right)\right\}^{2} S_{K}^{*} \\
& \leqslant \frac{1}{4} S_{K}^{*} \sum_{i=1}^{4} u_{h}^{2}\left(a_{i, K}\right) \leqslant \frac{C_{0}}{c_{0}} F_{2}\left(u_{h}^{2}\right) .
\end{aligned}
$$

Thus, (3.10) holds with $C=\left(C_{0} / c_{0}\right)^{3 / 2}$. The proof of (3.11) is obvious. It is easy to show that $[\cdot]_{h}$ (respectively $[\cdot]_{\Gamma_{h}}$ ) is not a norm on $V_{h}$ (respectively $V_{h}^{\prime}$ ).

The next lemma follows easily from (3.10) and $H 2$ :

LEMMA 3.3. There exists a constant $M$, independent of $h$, such that

$$
\left|a_{h}\left(u_{h}, v_{h}\right)\right| \leqslant M\left\|u_{h}\right\|_{h}\left\|v_{h}\right\|_{h} \quad \forall u_{h} \in V_{h}+V \text { and } \forall v_{h} \in V_{h} \text {. }
$$

LEMMA 3.4. If $\left\|b_{i}\right\|_{L^{\infty}(\widetilde{\Omega})}(i=1,2)$ are small enough, there exists a constant $\beta>0$, independent of $h$, such that

$$
a_{h}\left(v_{h}, v_{h}\right) \geqslant \beta\left\|v_{h}\right\|_{h}^{2} \quad \forall v_{h} \in V_{h} .
$$


Proof.

$$
\begin{aligned}
a_{h}\left(v_{h}, v_{h}\right)= & \sum_{K^{*} \in Q_{h}}\left(\left\{a_{11}\left(B_{K}\right) \frac{\partial v_{h}}{\partial x}\left(B_{K}\right)+a_{12}\left(B_{K}\right) \frac{\partial v_{h}}{\partial y}\left(B_{K}\right)\right\} \frac{\partial v_{h}}{\partial x}\left(B_{K}\right)\right. \\
& \left.+\left\{a_{21}\left(B_{K}\right) \frac{\partial v_{h}}{\partial x}\left(B_{K}\right)+a_{22}\left(B_{K}\right) \frac{\partial v_{h}}{\partial y}\left(B_{K}\right)\right\} \frac{\partial v_{h}}{\partial y}\left(B_{K}\right)\right) S_{K}^{*} \\
+ & {\left[b_{1} \frac{\partial v_{h}}{\partial x}+b_{2} \frac{\partial v_{h}}{\partial y}, v_{h}\right]_{h}+\left(d v_{h}, v_{h}\right)_{h}, } \\
a_{h}\left(v_{h}, v_{h}\right) \geqslant & \gamma \sum_{K} \in Q_{h}\left\{\frac{\partial v_{h}^{2}}{\partial x}\left(B_{K}\right)+\frac{\partial v_{h}^{2}}{\partial y}\left(B_{K}\right)\right\} S_{K}^{*}+\gamma\left|v_{h}\right|_{h}^{2} \\
& -C\left\{\left\|b_{1}\right\|_{L^{\infty}(\widetilde{\Omega})}\left[\frac{\partial v_{h}}{\partial x}\right]_{h}+\left\|b_{2}\right\|_{L}(\tilde{\Omega})\left[\frac{\partial v_{h}}{\partial y}\right]_{h}\right\}\left|v_{h}\right|_{h},
\end{aligned}
$$

thanks to $H 1, H 2$ and (3.10). Hence

$$
a_{h}\left(v_{h}, v_{h}\right) \geqslant \gamma\left\|v_{h}\right\|_{h}^{2}-C\left(\left\|b_{1}\right\|_{L^{\infty}(\tilde{\Omega})}+\left\|b_{2}\right\|_{L^{\infty}(\tilde{\Omega})}\right)\left\|v_{h}\right\|_{h}^{2} .
$$

If, for instance, $\left\|b_{1}\right\|_{L^{\infty}(\widetilde{\Omega})}+\left\|b_{2}\right\|_{L^{\infty}(\widetilde{\Omega})} \leqslant \gamma / 2 C$, then $a_{h}\left(v_{h}, v_{h}\right) \geqslant \gamma\left\|v_{h}\right\|_{h}^{2} / 2$.

LEMMA 3.5. Let $L_{h}\left(v_{h}\right)$ be defined by (3.9) or (3.9'). There exist two constants $\lambda_{1}$ and $\lambda_{2}$, independent of $h$, such that

$$
\left|L_{h}\left(v_{h}\right)\right| \leqslant \lambda_{1}\left|v_{h}\right|_{h}+\lambda_{2}\left|v_{h}\right|_{\Gamma_{h}} \forall v_{h} \in V_{h} .
$$

Proof. Let, for instance, $L_{h}\left(v_{h}\right)$ be defined by (3.9). Then it follows from (3.10), (3.11) and $\mathrm{H} 2$ that

$$
\left|L_{h}\left(v_{h}\right)\right| \leqslant\|f\|_{C^{0}(\tilde{\Omega})} C(\operatorname{mes} \widetilde{\Omega})^{1 / 2}\left|v_{h}\right|_{h}+\|g\|_{C^{0}(\Gamma)}(\operatorname{mes} \Gamma)^{1 / 2}\left|v_{h}\right|_{\Gamma_{h}} .
$$

We have thus shown that $a_{h}$ satisfies (2.2) and (2.3). However, Lemma 3.5 does not suffice to prove that $L_{h}$ satisfies (2.4). To achieve this, we must find an estimate for $\left|v_{h}\right|_{\Gamma_{h}}$ in terms of $\left\|v_{h}\right\|_{h}$. This is the object of Section 5 .

Remark 3.4. To guarantee that $a_{h}$ is positive definite, it is necessary to integrate the expression $d u_{h} v_{h}$ by formula $F_{2}$.

3.3. The Projection Error. This section gives estimates for $\inf _{v_{h} \in V_{h}}\left\|u-v_{h}\right\|_{h}$. We shall use the following seminorm for $u$ in $H^{m}(\Omega)$ :

$$
|u|_{m, 2, \Omega}=\left(\sum_{\alpha_{1}+\alpha_{2}=m}\left\|\frac{\partial^{m} u}{\partial x^{\alpha_{1}} \partial^{\alpha_{2}}}\right\|_{L^{2}(\Omega)}^{2}\right)^{1 / 2} .
$$

LEMMA 3.6. Let $u$ be defined on $\widetilde{\Omega}$ and let $r_{h} u$ be the "projection" of $u$ on $V_{h}$, as in subsection 3.1. We assume that $Q_{h}$ is locally regular.

$\left(1^{\circ}\right)$ If $u \in H^{3}(\Omega)$, there exists a constant $C$, independent of $u$ and $h$, such that,

$$
\left\|u-r_{h} u\right\|_{h} \leqslant C h\left(|u|_{2,2, \tilde{\Omega}}^{2}+h^{2}|u|_{3,2, \tilde{\Omega}}^{2}\right)^{1 / 2} .
$$

$\left(2^{\circ}\right)$ If $u$ belongs only to $V=H^{2}(\widetilde{\Omega}) \cap C^{1}(\widetilde{\Omega})$, then 


$$
\left\|u-r_{h} u\right\|_{h} \leqslant C^{\prime} h|u|_{2,2, \widetilde{\Omega}}
$$

where $C^{\prime}$ is another constant independent of $u$ and $h$.

Proof. Since $\left|u-r_{h} u\right|_{h}=0$, it suffices to consider

$$
\left[\frac{\partial}{\partial x}\left(u-r_{h} u\right)\right]_{h} \text { and }\left[\frac{\partial}{\partial y}\left(u-r_{h} u\right)\right]_{h} .
$$

Let $u \in H^{3}(\widetilde{\Omega})$. Let $K^{*}$ be a cell of $Q_{h}$, let $\hat{u}=u \circ F_{K}$, and let $F_{K}^{(1)}$ and $F_{K}^{(2)}$ denote the components of $F_{K}$. Then

$$
\begin{aligned}
\left\{\frac{\partial}{\partial x}\left(u-r_{h} u\right)\right\}\left(B_{K}\right) & \\
= & \frac{1}{S_{K^{*}}}\left\{\frac{\partial}{\partial \hat{x}}\left(\hat{a}-\widehat{\left.r_{h} u\right)} \frac{\partial}{\partial \hat{y}} F_{K}^{(2)}-\frac{\partial}{\partial \hat{y}}\left(\hat{u}-\widehat{r_{h} \hat{u}}\right) \frac{\partial}{\partial \hat{x}} F_{K}^{(2)}\right\}(\hat{B}),\right.
\end{aligned}
$$

and a similar equality holds for the derivative with respect to $y$. With the notations of Figure 2,

$$
\left(\frac{\partial}{\partial \hat{x}} \widehat{r_{h} u}\right)(\hat{B})=\frac{1}{2}\left[\left(\hat{u}_{3}-\hat{u}_{4}\right)+\left(\hat{u}_{2}-\hat{u}_{1}\right)\right] .
$$

Thus, the mapping $\hat{u} \mapsto\left[\partial\left(\hat{u}-\widehat{r_{h} \hat{u}}\right) / \partial \hat{x}\right](\hat{B})$ is a linear and continuous form on $H^{3}(\hat{K})$ which vanishes for polynomials of degree 2 . Therefore, by the Bramble and Hilbert lemma (cf. Bramble \& Hilbert [1]), there exists a constant $c$, independent of $u$ and $h$, such that

$$
\mid\left\{\left.\frac{\partial}{\partial \hat{x}}\left(\hat{u}-\widehat{\left.r_{h} \hat{u}\right)}\right\}(\hat{B})|\leqslant c| \hat{u}\right|_{3,2, \hat{K}}\right.
$$

Now, since $Q_{h}$ is locally regular,

$$
|\hat{u}|_{3,2, \mathbb{R}}^{2} \leqslant \frac{c^{\prime}}{c_{0}} h^{2}\left(|u|_{2,2, K^{*}}^{2}+h^{2}|u|_{3,2, K^{*}}^{2}\right),
$$

where $c^{\prime}$ is another constant independent of $u$ and $h$. As there is no loss of generality in having considered derivatives with respect to the first variable, (3.12) follows from these last inequalities, (3.14) and (3.6).

The proof of (3.13) is much the same as that of (3.12).

Hence, if $Q_{h}$ is locally regular, then, for fixed $u$ in $V$ :

$$
\inf _{v_{h} \in V_{h}}\left\|u-v_{h}\right\|_{h} \leqslant C h,
$$

where $C$ is a constant independent of $h$.

Remark 3.5. When $K^{*}$ is a parallelogram, the coefficient of $|u|_{2,2, K^{*}}^{2}$ in the right-hand side of (3.15) vanishes, and if $K^{*}$ deviates slightly from a parallelogram, this coefficient is small. More precisely, if

$$
\left\|a_{1, K}+a_{3, K}-a_{2, K}-a_{4, K}\right\|=O\left(h^{2}\right)
$$

then

$$
|\hat{u}|_{3,2, \hat{K}}^{2} \leqslant c h^{4}\left(|u|_{2,2, K^{*}}^{2}+|u|_{3,2, K^{*}}^{2}\right)
$$


where again $c$ is a constant independent of $u$ and $h$. If, moreover, $u \in \mathcal{C}^{3}(\widetilde{\Omega})$, then $\left[\partial\left(u-r_{h} u\right) / \partial x_{i}\right]\left(B_{K}\right)=O\left(h^{2}\right), i=1,2$, where $x_{i}$ denotes either $x$ or $y$. It is interesting to note that this last property has a converse stated in the next lemma.

LEMMA 3.7. Let $Q_{h}$ be locally regular and let $K^{*} \in Q_{h}$. If, for all functions $u$ in $C^{3}(\widetilde{\Omega}),\left\{\partial\left(u-r_{h} u\right) / \partial x_{i}\right\}\left(B_{K}\right)=O\left(h^{2}\right)$, for $i=1,2$, then $K^{*}$ is nearly a parallelogram, that is, its vertices satisfy (3.17).

The proof of this lemma is straightforward.

\section{Study of the Approximation.}

4.1. Notations Concerning the Quadrangulation. Let $\dot{O}_{h}$ denote the set of vertices of $Q_{h}$ and $\dot{\Gamma}_{h}$ the set of vertices of $\Gamma_{h}$. Let $\dot{\Omega}_{h}=\dot{O}_{h}-\dot{\Gamma}_{h}$. According to hypothesis (3.4), we can number the nodes of $\dot{O}_{h}$ with a pair of indices $(i, j)$. This numbering induces obviously another one on the cells $K^{*}$ of $Q_{h}$ (cf. Figure 2). Let $\dot{\Omega}_{h}^{*}=$ mean center of $K^{*} \mid K^{*} \in 2_{h}$ \}. If $(i, j)$ is the index of $K^{*}$, we agree to denote this cell by $K_{i, j}^{*}$ and its area by $S_{i, j}^{*}$. Also, it will be convenient to denote its mean center by $(i, j)$ and by $\varphi_{i, j}$ the value of a function $\varphi$ at $(i, j)$. Similarly, if $(i, j)$ is the index of a node of $\dot{O}_{h}$, we agree to denote by $\varphi_{i, j}$ the value of $\varphi$ at $(i, j)$.

Now, let $(i, j)$ be a node of $\dot{O}_{h}$, let $R_{i, j} \subset \Omega_{h}^{*}$ denote the region consisting of the triangles of $\Omega_{h}^{*}$ with common vertex $(i, j)$ and let $B_{i, j}$ stand for the boundary of $R_{i, j}$ (cf. Figure 3). Let

$$
S_{i, j}=1 / 2\left(\text { area of } R_{i, j}\right)
$$

and, for $(i, j)$ in $\dot{\Gamma}_{h}$, let $l_{i, j}=1 / 2\left(\right.$ length of $\left.B_{i, j} \cap \Gamma_{h}\right)$.

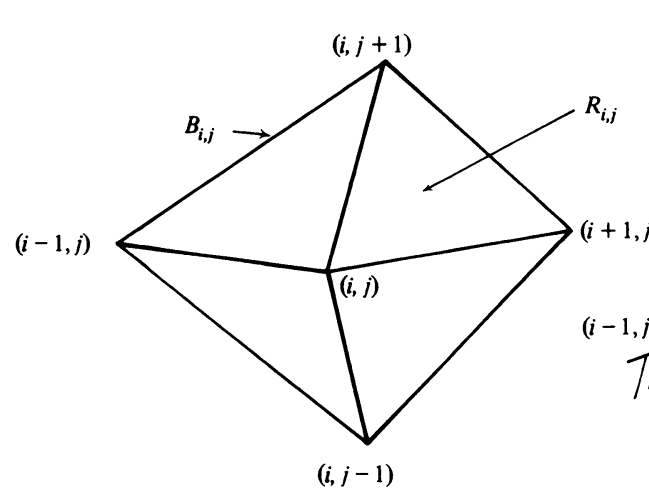

(a)

FIGURE 3

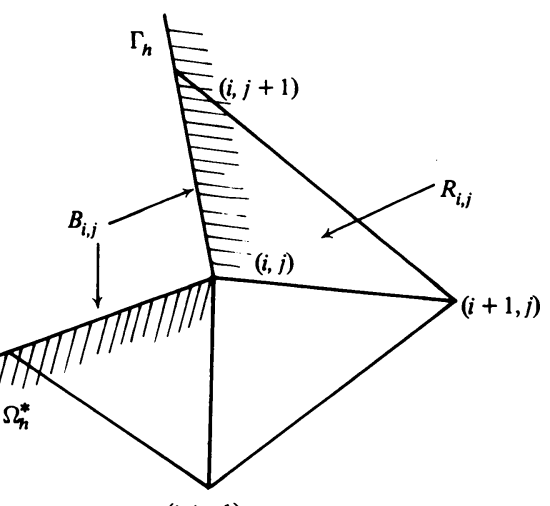

$(i, j-1)$

(b)

With these notations, we can express easily the scalar products $(\cdot,)_{h}$ and $(\cdot, \cdot)_{\Gamma_{h}}$

LEMMA 4.1.

$$
\left.\begin{array}{rl}
\left(u_{h}, v_{h}\right)_{h} & =\sum_{(i, j) \in \dot{O}_{h}} u_{i, j} v_{i, j} s_{i, j} \\
\left(u_{h}, v_{h}\right)_{\Gamma_{h}} & =\sum_{(i, j) \in \dot{\Gamma}_{h}} u_{i, j} v_{i, j} l_{i, j}
\end{array}\right\} \forall u_{h}, v_{h} \in V_{h} .
$$

Remark 4.1. It follows from Remark 3.2 that 


$$
\begin{aligned}
& c_{0} h^{2} \leqslant S_{i, j} \leqslant C_{0} h^{2} \\
& \frac{c_{0}}{4} h^{2} \leqslant S_{i, j} \leqslant \frac{3}{4} C_{0} h^{2} \\
& \sigma_{0} h \leqslant l_{i, j} \leqslant h
\end{aligned} \quad \forall(i, j) \in \dot{\Omega}_{h},
$$

Finally, let $V_{h}^{*} \subset L^{2}\left(\Omega_{h}^{*}\right)$ denote the space of real step functions which are constant on each cell of $Q_{h}$. With each real function $\varphi$ defined on $\dot{\Omega}_{h}^{*}$, we associate the function $r_{h}^{*} \varphi$ of $V_{h}^{*}$ which takes the same value as $\varphi$ at the nodes of $\dot{\Omega}_{h}^{*}$.

\subsection{Difference Quotients and Averages.}

Definition 4.1. Let $\varphi$ be a function in $V_{h}^{*}$. We define its discrete derivative with respect to $x$ as follows:

$$
\left(\nabla_{1}^{*} \varphi\right)_{i, j}=\frac{1}{2 S_{i, j}} \oint_{B_{i, j}} \varphi(x, y) d y \quad \forall(i, j) \in \dot{O}_{h},
$$

and its discrete derivative with respect to $y$ by:

$$
\left(\nabla_{2}^{*} \varphi\right)_{i, j}=-\frac{1}{2 S_{i, j}} \oint_{B_{i, j}} \varphi(x, y) d x \quad \forall(i, j) \in \dot{O}_{h}
$$

It can be easily checked that, for $(i, j)$ in $\dot{\Omega}_{h}$,

$$
\begin{aligned}
\left(\nabla_{1}^{*} \varphi\right)_{i, j}=\frac{1}{2 S_{i, j}}\left\{\varphi_{i+1 / 2, j+1 / 2}\left(y_{i, j+1}-y_{i+1, j}\right)+\varphi_{i-1 / 2, j+1 / 2}\left(y_{i-1, j}-y_{i, j+1}\right)\right. \\
\left.+\varphi_{i-1 / 2, j-1 / 2}\left(y_{i, j-1}-y_{i-1, j}\right)+\varphi_{i+1 / 2, j-1 / 2}\left(y_{i+1, j}-y_{i, j-1}\right)\right\} \\
\left(\nabla_{2}^{*} \varphi\right)_{i, j}=\frac{1}{2 S_{i, j}}\left\{\varphi_{i+1 / 2, j+1 / 2}\left(x_{i+1, j}-x_{i, j+1}\right)+\varphi_{i-1 / 2, j+1 / 2}\left(x_{i, j+1}-x_{i-1, j}\right)\right. \\
\left.+\varphi_{i-1 / 2, j-1 / 2}\left(x_{i-1, j}-x_{i, j-1}\right)+\varphi_{i+1 / 2, j-1 / 2}\left(x_{i, j-1}-x_{i+1, j}\right)\right\}
\end{aligned}
$$

These formulas are, of course, different when $(i, j) \in \dot{\Gamma}_{h}$. As an example, if $(i, j)$ is located as in Figure 3(b), then (4.5) yields:

$$
\begin{aligned}
\left(\nabla_{1}^{*} \varphi\right)_{i, j}=\frac{1}{2 S_{i, j}}\left\{\varphi_{i-1 / 2, j-1 / 2}\left(y_{i, j-1}-y_{i, j}\right)+\right. & \varphi_{i+1 / 2, j-1 / 2}\left(y_{i+1, j}-y_{i, j-1}\right) \\
& \left.+\varphi_{i+1 / 2, j+1 / 2}\left(y_{i, j}-y_{i+1, j}\right)\right\} .
\end{aligned}
$$

The usefulness of these difference quotients appears in the following result:

Proposition 4.1. Let $\varphi$ be a function defined on $\Omega_{h}^{*}$ and $v_{h}$ a function of $V_{h}$. Then the following "discrete Green's formula" holds:

$$
\left[\varphi, \frac{\partial v_{h}}{\partial x_{i}}\right]_{h}=-\left(r_{h}\left(\nabla_{i}^{*} \varphi\right), v_{h}\right)_{h}+\left[r_{h}^{*} \varphi \cdot \cos \left(\vec{n}_{h}, x_{i}\right), v_{h}\right]_{\Gamma_{h}}, \quad i=1,2,
$$

where $x_{i}$ denotes either $x$ or $y$ and $\cos \left(\vec{n}_{h}, x_{i}\right)$ denotes the ith direction cosine of the exterior normal to $\Gamma_{h}$.

The proof is omitted since it is rather long and technical. 
Definition 4.2. Let $\varphi$ be a function in $V_{h}^{*}$. We define its average $\mu^{*} \varphi$ as follows:

$$
\left(\mu^{*} \varphi\right)_{i, j}=\frac{1}{4 S_{i, j}} \sum_{(r, s) \in \dot{\Omega}_{h}^{*} ; r=i \pm 1 / 2 ; s=j \pm 1 / 2} \varphi_{r, s} S_{r, s}^{*} \quad \forall(i, j) \in \dot{O}_{h}
$$

This formula yields, when $(i, j) \in \dot{\Omega}_{h}$ :

$$
\begin{aligned}
\left(\mu^{*} \varphi\right)_{i, j}=\frac{1}{4 S_{i, j}}\{ & \varphi_{i+1 / 2, j+1 / 2} S_{i+1 / 2, j+1 / 2}^{*}+\varphi_{i-1 / 2, j+1 / 2} S_{i-1 / 2, j+1 / 2}^{*} \\
& \left.+\varphi_{i-1 / 2, j-1 / 2} S_{i-1 / 2, j-1 / 2}^{*}+\varphi_{i+1 / 2, j-1 / 2} S_{i+1 / 2, j-1 / 2}^{*}\right\}
\end{aligned}
$$

If $(i, j) \in \dot{\Gamma}_{h}$, as in Figure 3(b) for instance, formula (4.10) becomes:

$$
\begin{aligned}
\left(\mu^{*} \varphi\right)_{i, j}=\frac{1}{4 S_{i, j}}\left\{\varphi_{i-1 / 2, j-1 / 2} S_{i-1 / 2, j-1 / 2}^{*}\right. & +\varphi_{i+1 / 2, j-1 / 2} S_{i+1 / 2, j-1 / 2}^{*} \\
& \left.+\varphi_{i+1 / 2, j+1 / 2} S_{i+1 / 2, j+1 / 2}^{*}\right\} .
\end{aligned}
$$

The averaging operator $\mu^{*}$ is related to $V_{h}$ in the following way:

LEMma 4.2. Let $\varphi$ be defined on $\Omega_{h}^{*}$ and let $v_{h}$ belong to $V_{h}$, then

$$
\left[\varphi, v_{h}\right]_{h}=\left(r_{h}\left(\mu^{*} \varphi\right), v_{h}\right)_{h} \text {. }
$$

The proof is much like that of Proposition 4.1.

4.3. Some Properties of Difference Quotients and Averages. Regularity Conditions on $2_{n}$. The next lemma follows easily from the regularity hypothesis $\mathrm{H} 3$ on $\Gamma$ :

Lemma 4.3. Let $\varphi \in C^{1}(\widetilde{\Omega})$ and $v_{h} \in V_{h}^{\prime}$. Then, for $i=1,2$,

$$
\left[r_{h}^{*} \varphi \cdot \cos \left(\vec{n}_{h}, x_{i}\right), v_{h}\right]_{\Gamma_{h}}=\left[\varphi \cos \left(\vec{n}, x_{i}\right), v_{h}\right]_{\Gamma_{h}}+O(h)\left|v_{h}\right|_{\Gamma_{h}}
$$

Besides the local regularity condition (3.1) (3.2), let us introduce two additional regularity conditions on $2 h$.

Definitions 4.3. $\left(1^{\circ}\right)$ We say that quadrangulation $Q_{h}$ is globally regular if, at every node $(i, j)$ of $\dot{\Omega}_{h}$, the following holds:

$$
\left(\nabla_{k}^{*} x_{l}\right)_{i, j}=\delta_{k l}+O(h) \text { for } k, l=1,2 .
$$

(2 $\left.2^{\circ}\right) 2_{h}$ is said to be regular in the mean if, at each node $(i, j)$ of $\dot{\Omega}_{h}$, we have

$$
\begin{aligned}
S_{i, j}= & \frac{1}{4}\left\{S_{i+1 / 2, j+1 / 2}^{*}+S_{i-1 / 2, j+1 / 2}^{*}+S_{i-1 / 2, j-1 / 2}^{*}+S_{i+1 / 2, j-1 / 2}^{*}\right\} \\
& +O\left(h^{3}\right) . \square
\end{aligned}
$$

Proposition 4.2. Let $\varphi \in C^{2}(\widetilde{\Omega})$. If $Q_{h}$ is globally regular, the following equality holds at each node $(i, j)$ of $\dot{\Omega}_{h}$ :

$$
\left(\nabla_{k}^{*} \varphi\right)_{i, j}=\left(\partial \varphi / \partial x_{k}\right)_{i, j}+O(h), \quad k=1,2
$$

The proof of this proposition is straightforward (cf. Girault [4]).

LEMMA 4.4. Let $\varphi \in C^{1}(\widetilde{\Omega})$ and $v_{h} \in V_{h}$. If $Q_{h}$ is regular in the mean, we have

$$
\left[\varphi, v_{h}\right]_{h}=\left(\varphi, v_{h}\right)_{h}+O(h)\left|v_{h}\right|_{h}+O(h)\left|v_{h}\right|_{\Gamma_{h}} \text {. }
$$

Proof. According to Lemma 4.2, we must study $\mu^{*} \varphi$. Let $(i, j) \in \dot{\Omega}_{h}$ and let us apply (4.11); we get 


$$
\begin{aligned}
\left(\mu^{*} \varphi\right)_{i, j}=\frac{1}{4 S_{i, j}}\left\{S_{i+1 / 2, j+1 / 2}^{*}+S_{i-1 / 2, j+1 / 2}^{*}\right. & \\
& \left.+S_{i-1 / 2, j-1 / 2}^{*}+S_{i+1 / 2, j-1 / 2}^{*}\right\}\left(\varphi_{i, j}+O(h)\right)
\end{aligned}
$$

Since $2_{h}$ is regular in the mean, this implies that

$$
\left(\mu^{*} \varphi\right)_{i, j}=\left(\varphi_{i, j}+O(h)\right)(1+O(h)) .
$$

Now, let $(i, j) \in \dot{\Gamma}_{h}$; for instance, let us consider the case of Figure $3(\mathrm{~b})$, then

$$
\left(\mu^{*} \varphi\right)_{i, j}=\frac{1}{4 S_{i, j}}\left\{S_{i-1 / 2, j-1 / 2}^{*}+S_{i+1 / 2, j-1 / 2}^{*}+S_{i+1 / 2, j+1 / 2}^{*}\right\}\left(\varphi_{i, j}+O(h)\right) .
$$

No assumption has been made on the quotient of areas in the neighborhood of $\Gamma_{h}$, but by virtue of (3.6) and (4.3'), we find that

$$
\frac{1}{4 S_{i, j}}\left[S_{i-1 / 2, j-1 / 2}^{*}+S_{i+1 / 2, j-1 / 2}^{*}+S_{i+1 / 2, j+1 / 2}^{*}\right] \leqslant \frac{C_{0}}{c_{0}} \text {. }
$$

Obviously, this last inequality holds at any point of $\dot{\Gamma}_{h}$. Hence,

$$
\left(\mu^{*} \varphi\right)_{i, j}=\varphi_{i, j}+\rho_{i, j} \quad \forall(i, j) \in \dot{\Gamma}_{h},
$$

where $\left|\rho_{i, j}\right| \leqslant C$ and $C$ is independent of $h$ and $(i, j)$. Thus, thanks to (4.12) and (4.1),

$$
\begin{aligned}
{\left[\varphi, v_{h}\right]_{h} } & =\sum_{(i, j) \in \dot{O}_{h}}\left(\mu^{*} \varphi\right)_{i, j} v_{i, j} S_{i, j} \\
& =\sum_{(i, j) \in \dot{O}_{h}} \varphi_{i, j} v_{i, j} S_{i, j}+\sum_{(i, j) \in \dot{\Omega}_{h}} O(h) v_{i, j} S_{i, j}+\sum_{(i, j) \in \dot{\Gamma}_{h}} \rho_{i, j} v_{i, j} S_{i, j}
\end{aligned}
$$

Hence $\left[\varphi, v_{h}\right]_{h}=\left(\varphi, v_{h}\right)_{h}+O(h)\left|v_{h}\right|_{h}+R$, where

$$
|R|=\left|\sum_{(i, j) \in \dot{\Gamma}_{h}} \rho_{i, j} v_{i, j} s_{i, j}\right|
$$

Now, by virtue of $\left(4.3^{\prime}\right)$ and $(4.4)$,

$$
|R| \leqslant C \cdot \frac{3}{4} \frac{C_{0} h}{\sigma_{0}} \sum_{(i, j) \in \dot{\Gamma}_{h}}\left|v_{i, j}\right| l_{i, j}
$$

Therefore, $R=O(h)\left|v_{h}\right|_{\Gamma_{h}}$.

In the sequel, we shall assume that besides satisfying hypotheses (3.1) to (3.4), $Q_{h}$ is also globally regular and regular in the mean.

LEMmA 4.5. Let $\varphi \in \mathcal{C}^{2}(\widetilde{\Omega})$ and $v_{h} \in V_{h}$. Then, for $i=1,2$,

$$
\left(r_{h}\left(\nabla_{i}^{*} \varphi\right), v_{h}\right)_{h}=\left(\frac{\partial \varphi}{\partial x_{i}}, v_{h}\right)_{h}+O(h)\left|v_{h}\right|_{h}+O(h)\left|v_{h}\right|_{\Gamma_{h}} .
$$

The proof, which makes use of Proposition 4.2, is similar to that of the preceding lemma.

We derive the next proposition on combining Proposition 4.1 with Lemmas 4.3 and 4.5. 
Proposition 4.3. Let $\varphi \in \mathcal{C}^{2}(\widetilde{\Omega})$ and $v_{h} \in V_{h}$. Then, for $i=1,2$,

$$
\begin{aligned}
{\left[\varphi, \frac{\partial v_{h}}{\partial x_{i}}\right]_{h}=} & -\left(\frac{\partial \varphi}{\partial x_{i}}, v_{h}\right)_{h}+\left[\varphi \cos \left(\vec{n}, x_{i}\right), v_{h}\right]_{\Gamma_{h}} \\
& +O(h)\left|v_{h}\right|_{h}+O(h)\left|v_{h}\right|_{\Gamma_{h}} \cdot \square
\end{aligned}
$$

\section{Boundary Value Theorem.}

5.1. An Upper Bound. Let $K$ be a cell of $Q_{h}$ with mean center $B_{K}$, as in Figure 2. For the sake of simplicity, we denote by $\varphi_{i}$ the value of a function $\varphi$ at the vertex $a_{i, K}$ of $K$.

LEMMA 5.1. Let $v_{h} \in V_{h}$. With the above notations, we have

$$
\begin{aligned}
& v_{1}=v_{3}+\left(x_{1}-x_{3}\right) \frac{\partial v_{h}}{\partial x}\left(B_{K}\right)+\left(y_{1}-y_{3}\right) \frac{\partial v_{h}}{\partial y}\left(B_{K}\right), \\
& v_{2}=v_{4}+\left(x_{2}-x_{4}\right) \frac{\partial v_{h}}{\partial x}\left(B_{K}\right)+\left(y_{2}-y_{4}\right) \frac{\partial v_{h}}{\partial y}\left(B_{K}\right) .
\end{aligned}
$$

Now, let $N$ be a positive integer and $r$ an integer such that $0 \leqslant r \leqslant N-1$. Let us introduce a function of $V_{h}, \theta_{h, r}$, defined on $K$ by:

$$
\left(\theta_{h, r}\right)_{2}=\left(\theta_{h, r}\right)_{3}=1-\frac{r+1}{N}, \quad\left(\theta_{h, r}\right)_{1}=\left(\theta_{h, r}\right)_{4}=1-\frac{r}{N},
$$

and, for $r \geqslant 1$, a function of $V_{h}, \bar{\theta}_{h, r}$, which coincides with $\theta_{h, r}$ at the vertices of $K$ except at node $a_{1, K}$ (or $\left.a_{4, K}\right)$ where $\bar{\theta}_{h, r}=1-(r-1) / N$. This type of function has already been used by Cea (cf. Cea [2]) in the proof of boundary value theorems in the case of finite differences on regular meshes.

Lemмa 5.2. Let $v_{h} \in V_{h}$ and $0 \leqslant r \leqslant N-1$. Then, for $i=1,2$,

$$
\left.\begin{array}{l}
\left|\left[\frac{\partial}{\partial x_{i}}\left(\theta_{h, r} v_{h}^{2}\right)\right]\left(B_{K}\right)\right| \mid<\frac{3}{4 N c_{0} h}\left(v_{1}^{2}+v_{2}^{2}+v_{3}^{2}+v_{4}^{2}\right) \\
\left|\left[\frac{\partial}{\partial x_{i}}\left(\bar{\theta}_{h, r} v_{h}^{2}\right)\right]\left(B_{K}\right)\right|
\end{array}\right\} \begin{aligned}
& +\frac{1}{2}\left(\left|v_{1}\right|+\left|v_{2}\right|+\left|v_{3}\right|+\left|v_{4}\right|\right)\left|\frac{\partial v_{h}}{\partial x_{i}}\left(B_{K}\right)\right| \quad(r \neq 1) .
\end{aligned}
$$

Proof. The lemma follows immediately from the fact that

$$
\begin{gathered}
\bar{\theta}_{h, r}\left(B_{K}\right)<\theta_{h, r}\left(B_{K}\right)<1, \\
\left|\frac{\partial}{\partial x_{i}} \theta_{h, r}\left(B_{K}\right)\right| \leqslant \frac{2}{N c_{0} h} \text { and }\left|\frac{\partial}{\partial x_{i}} \bar{\theta}_{h, r}\left(B_{K}\right)\right| \leqslant \frac{3}{N c_{0} h} \quad(r \neq 1) .
\end{gathered}
$$

Definitions 5.1. $\left(1^{\circ}\right)$ Let $M$ be a vertex of $Q_{h}$ and $k$ a positive integer. An elementary path of length $k$ and origin $M$ in $Q_{h}$ is a broken oriented line, without double point, starting at $M$, and consisting of $k$ consecutive and distinct diagonals of cells of $2_{n}$. The endpoints of these diagonals are called vertices of the path. We agree to number the vertices from 0 to $k$, beginning with $M$.

$\left(2^{\circ}\right)$ A string of length $k$ in $Q_{h}$ is an ordered sequence of $k$ distinct cells of $Q_{h}$, $K_{1}, K_{2}, \ldots, K_{k}$, such that $K_{i}$ and $K_{i+1}$ share a common side or vertex, for $1 \leqslant i \leqslant$ $k-1$. 
Remark 5.1. The set of cells crossed by an elementary path of length $k$ and ordered in a natural fashion by the orientation of the path forms a string of length $k$.

Lemma 5.3. Let $v_{h} \in V_{h}$. Let $M$ be a vertex of $2_{h}, N$ a positive integer and $P$ an elementary path of length $N$ and origin $M$ in $2_{n}$. Let $K_{0} K_{1} \cdots K_{N-1}$ be the string of cells crossed by $P$ and let $\left(i_{p}, j_{p}\right)$ be the indices of the mean center of $K_{p}$. Then,

$$
\begin{aligned}
v_{h}^{2}(M) \leqslant h \sum_{p=0}^{N-1}\left\{\left(\sum_{r=i_{p} \pm 1 / 2 ; s=j_{p} \pm 1 / 2}\left|v_{r, s}\right|\right)\left(\left|\frac{\partial v_{h}}{\partial x}\right|_{i_{p}, j_{p}}+\left|\frac{\partial v_{h}}{\partial y}\right|_{i_{p}, j_{p}}\right)\right. \\
\left.+\frac{3}{N c_{0} h}\left(\sum_{r=i_{p} \pm 1 / 2 ; s=j_{p} \pm 1 / 2} v_{r, s}^{2}\right)\right\} .
\end{aligned}
$$

Proof. Let $M_{0}=M, M_{1}, M_{2}, \ldots, M_{N}$ denote the vertices of $P$ and let $\Theta_{h}$ be a function of $V_{h}$ defined on $K_{0}, K_{1}, \ldots, K_{N-1}$ by:

$$
\left.\begin{array}{l}
\Theta_{h}\left(M_{r}\right)=1-r / N \text { and } \\
\Theta_{h}=\theta_{h, r} \text { or } \Theta_{h}=\bar{\theta}_{h, r} \text { on } K_{r}
\end{array}\right\}, \quad 0 \leqslant r \leqslant N,
$$

where, for obvious continuity reasons, $\bar{\theta}_{h, r}$ is used instead of $\theta_{h, r}$ when $K_{r}, K_{r-1}$ and $K_{r-2}$ share a common vertex $(r \geqslant 2)$.

Let $w_{h}=v_{h}^{2} \Theta_{h}$. Then

$$
w_{h}\left(M_{0}\right)=v_{h}^{2}(M) \text { and } w_{h}\left(M_{N}\right)=0 .
$$

Applying Lemma 5.1 to $K_{p}$, for $0 \leqslant p \leqslant N-1$, we find:

$$
\left|w_{h}\left(M_{p}\right)\right| \leqslant\left|w_{h}\left(M_{p+1}\right)\right|+2 h\left(\left|\frac{\partial}{\partial x} w_{h}\right|_{i_{p}, j_{p}}+\left|\frac{\partial}{\partial y} w_{h}\right|_{i_{p}, j_{p}}\right) .
$$

It can be easily shown by induction that this inequality and (5.3) yield

$$
v_{h}^{2}(M) \leqslant 2 h \sum_{p=0}^{N-1}\left\{\left|\frac{\partial}{\partial x} w_{h}\right|_{i_{p}, j_{p}}+\left|\frac{\partial}{\partial y} w_{h}\right|_{i_{p}, j_{p}}\right\} .
$$

The desired result is obtained on substituting (5.1) in the last inequality.

5.2. Continuity of Boundary Values for Norm \|\|$_{h}$ of $V_{h}$.

Lemma 5.4. Let $C$ be one of the arcs of $\Gamma$. There exist a finite covering $c f C$, $\left\{\Gamma_{1}, \Gamma_{2}, \ldots, \Gamma_{\nu}\right\}$, a set of directions, $\left\{\vec{d}_{1}, \vec{d}_{2}, \ldots, \vec{d}_{\nu}\right\}$, and two positive numbers, $t$ and $\eta$ such that, for $1 \leqslant i \leqslant \nu$ :

$\left(1^{\circ}\right)$ the cylinder of length $t$ borne by $\Gamma_{i}$ in direction $\vec{d}_{i}$ is contained in $\Omega$; than $\eta$.

$\left(2^{\circ}\right) \Gamma_{i} \cap \Gamma_{i+1} \neq \varnothing \quad(i \neq v)$ and the length of the chord to $\Gamma_{i} \cap \Gamma_{i+1}$ is greater

Proof. Let $\theta_{0}$ be the angle of hypothesis $\mathrm{H} 3$ and let us choose an angle $\lambda$ such that $0<2 \lambda<\theta_{0}$. Let $\vec{f}:[a, b] \rightarrow C$ be the intrinsic parametric representation of $C$. According to $\mathrm{H} 3, \vec{f}^{\prime} \in C^{0}[a, b]$; hence, there exists a step $\Delta \tau>0$ such that: 
for any $\tau$ and $\tau^{*}$ in $[a, b],\left|\tau-\tau^{*}\right| \leqslant \Delta \tau$ implies that the angle between $\vec{f}^{\prime}(\tau)$ and $\vec{f}^{\prime}\left(\tau^{*}\right)$ is smaller than $1 / 2\left(\theta_{0} / 2-\lambda\right)$.

Let $\Delta \tau \leqslant(b-a) / 4$. We shall construct a finite covering of $[a, b]$ with overlapping intervals:

$$
\begin{aligned}
& \left\{\left[a, \tau_{1}\right],\left[\bar{\tau}_{1}, \tau_{2}\right], \ldots,\left[\bar{\tau}_{\nu-1}, b\right]\right\} \quad \text { such that } \\
& \tau_{k}-\bar{\tau}_{k}=\Delta \tau \text { for } 1 \leqslant k \leqslant \nu-1 .
\end{aligned}
$$

A direction $\vec{d}_{k}$ will be associated with each interval $\left[\bar{\tau}_{k-1}, \tau_{k}\right], 1 \leqslant k \leqslant \nu$. Let $\varphi_{k}(\tau)$ denote the angle between $\vec{d}_{k}$ and $\vec{f}^{\prime}(\tau)$ on $\left[\bar{\tau}_{k-1}, \tau_{k}\right]$. The covering and set of directions will be chosen so that

$$
\lambda<\varphi_{k}(\tau)<\pi-\lambda \text { on }\left[\bar{\tau}_{k-1}, \tau_{k}\right] \text { for } 1 \leqslant k \leqslant \nu .
$$

Let the first direction $\vec{d}_{1}$ (respectively, last direction $\vec{d}_{\nu}$ ) be that of the interior bisector to the angle between the tangents at the intersection of $\operatorname{arc} C$ and its preceding arc (respectively, following arc). Then

$$
\frac{\theta_{0}}{2} \leqslant \varphi_{1}(a) \leqslant \pi-\frac{\theta_{0}}{2} \text { and } \frac{\theta_{0}}{2} \leqslant \varphi_{\nu}(b) \leqslant \pi-\frac{\theta_{0}}{2} .
$$

Therefore,

$$
\lambda<\varphi_{1}(a)<\pi-\lambda \text { and } \lambda<\varphi_{1}(b)<\pi-\lambda \text {. }
$$

The choice of $\Delta \tau$ implies that the first inequality holds on an interval $\left[a, \tau_{1}^{\prime}\right]$ and the second on an interval $\left[b^{\prime}, b\right]$, where:

$$
\tau_{1}^{\prime}-a \geqslant 2 \Delta \tau \text { and } b-b^{\prime} \geqslant 2 \Delta \tau .
$$

- If $\tau_{1}^{\prime}<b^{\prime}+\Delta \tau$, we take $\tau_{1}=\tau_{1}^{\prime}, \bar{\tau}_{1}=\tau_{1}-\Delta \tau$, and we choose for $\vec{d}_{2}$ the direction of the interior normal to $C$ at $\bar{\tau}_{1}$. Then (5.5) certainly holds at $\bar{\tau}_{1}$ (since $\left.\varphi_{2}\left(\bar{\tau}_{1}\right)=\pi / 2\right)$, and we can repeat the process with starting point $\bar{\tau}_{1}$ and direction $\vec{d}_{2}$. Since the length of each interval is greater than $2 \Delta \tau$, we reach $b^{\prime}+\Delta \tau$ after a finite number of steps.

- If $\tau_{k}^{\prime} \geqslant b^{\prime}+\Delta \tau$, we end the process by taking $\nu=k+1, \tau_{\nu-1}=$ $\max \left(b^{\prime}+\Delta \tau, a+2 \Delta \tau\right)$ and $\bar{\tau}_{\nu-1}=\tau_{\nu-1}-\Delta \tau$.

By construction, this covering satisfies (5.4) and (5.5).

Now, let $\Gamma_{k}$ denote the image by $\vec{f}$ of $\left[\bar{\tau}_{k-1}, \tau_{k}\right], 1 \leqslant k \leqslant \nu$. Then, thanks to (5.5) and the choice of direction $\vec{d}_{k}$, there exists a number $t_{k}>0$ such that the cylinder of length $t_{k}$ borne by $\Gamma_{k}$ in direction $\vec{d}_{k}$ is contained in $\Omega$. Moreover, (5.4) implies that $\Gamma_{k} \cap \Gamma_{k+1}$ is an arc of $C$ with chord of positive length, say $\eta_{k}$, for $1 \leqslant k \leqslant v-1$. Since $\nu$ is finite, the lemma is proved with

$$
t=\min _{1 \leqslant k \leqslant \nu} t_{k} \text { and } \eta=\min _{1 \leqslant k \leqslant \nu-1} \eta_{k} .
$$

Remarks 5.2. ( $\left.1^{\circ}\right) \nu, t$ and $\eta$ depend upon $C$, but the angle $\lambda$ does not and may be chosen for the entire boundary $\Gamma$.

$\left(2^{\circ}\right)$ If $C$ is reasonably smooth, $\nu$ is small (cf. Figure 4). In particular, $\nu=2$ when $C$ is a straight line. 


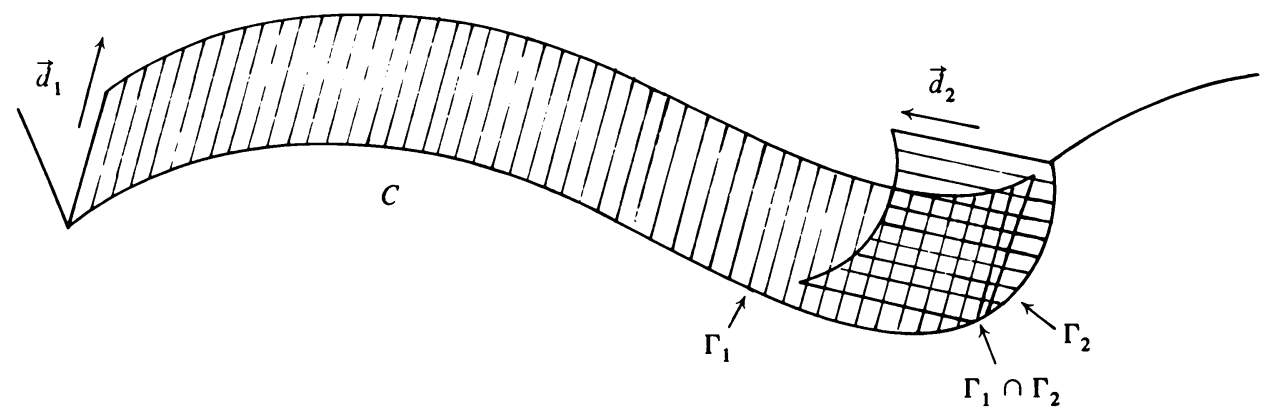

Figure 4

Since $\Gamma$ consists of a finite number of arcs, we derive the next theorem immediately from Lemma 5.4 .

THEOREM 5.1. There exist a finite covering of $\Gamma,\left\{\Gamma_{1}, \Gamma_{2}, \ldots, \Gamma_{n}\right\}$, a set of directions, $\left\{\vec{d}_{1}, \vec{d}_{2}, \ldots, \vec{d}_{n}\right\}$, and two positive numbers $l$ and $h_{0}$ such that, for $1 \leqslant i$ $\leqslant n$ :

$\left(1^{\circ}\right)$ the cylinder of length $l$ borne by $\Gamma_{i}$ in direction $\vec{d}_{i}$ is contained in $\Omega$;

$\left(2^{\circ}\right) \Gamma_{i} \cap \Gamma_{i+1} \neq \varnothing$ and, except at the angular points of $\Gamma$, the length of the chord to $\Gamma_{i} \cap \Gamma_{i+1}$ is greater than $h_{0}$.

COROLlARY 5.1. Let $h$ be small enough. Then, for each pair of adjacent nodes, $M$ and $P$, of $\dot{\Gamma}_{h}$, there exists a direction $\vec{d}$ belonging to the set $\left\{\vec{d}_{1}, \ldots, \vec{d}_{n}\right\}$ such that the parallelogram borne by $\overline{M P}$, with length $l / 2$ in direction $\vec{d}$, is contained in $\Omega_{h}^{*}$.

This corollary follows easily from Theorem 5.1 and the fact that $\Omega_{h}^{*}$ approximates $\Omega$.

From now on, we shall assume that $h$ is sufficiently small for Corollary 5.1 to hold. Note that, for most domains, this is not a severe restriction.

LEMMA 5.5. Let $M$ and $P$ be two adjacent nodes of $\dot{\Gamma}_{h}$ such that (for example) $\overrightarrow{M P}$ is oriented in the positive direction of integration. Let $T_{M}$ denote the parallelogram of Corollary 5.1. There exists an elementary path $P_{M}$ of length $N$ and origin $M$ such that:

- each cell of the string associated with $P_{M}$ cuts $T_{M}$;

Proof. We only sketch the proof. It proceeds in two steps:

$\left(1^{\circ}\right)$ We construct a string $K_{0} K_{1} \cdots K_{p}$ of cells such that each $K_{i}$ cuts $T_{M}, K_{i}$ and $K_{i+1}$ share a common side, $\overline{P M}$ is a side of $K_{0}$ and finally, the distance from $M$ to the center of $K_{p}$ is greater than $l / 2$. Denote by $K_{p}^{\prime}$ the side of $K_{p}$ opposite to the common side of $K_{p}$ and $K_{p-1}$.

$\left(2^{\circ}\right)$ We construct an elementary path $P_{M}$ with origin $M$, terminal at one of the extremities of $K_{p}^{\prime}$, and crossing a substring of $K_{0} K_{1} \cdots K_{p}$. Let $N$ be the length of $P_{M}$. By construction, each cell crossed by $P_{M}$ cuts $T_{M}$. Finally, since the length of each diagonal is smaller than $2 h$, we have $N h>l / 4$.

THEOREM 5.2. There exists a constant $C$, independent of $h$, such that

$$
\left|v_{h}\right|_{\Gamma_{h}} \leqslant C\left\|v_{h}\right\|_{h} \quad \forall v_{h} \in V_{h} .
$$


Proof. According to (4.2), $\left|v_{h}\right|_{\Gamma_{h}}^{2}=\Sigma_{M \in \dot{\Gamma}_{h}} v_{h}^{2}(M) l_{M}$.

Let $M$ be a node of $\dot{\Gamma}_{h}$. Let $T_{M}$ be the parallelogram and $P_{M}$ a path of origin $M$ satisfying the properties of the preceding lemma. Let $N$ be the length of $P_{M}$. Applying Lemma 5.3 and inequality (5.6), we get:

$$
\begin{aligned}
& v_{h}^{2}(M) l_{M} \leqslant h^{2} \sum_{p=0}^{N-1}\left\{\left(\sum_{r=i_{p} \pm 1 / 2 ; s=j_{p} \pm 1 / 2}\left|v_{r, s}\right|\right)\left(\left|\frac{\partial v_{h}}{\partial x}\right|_{i_{p}, j_{p}}+\left|\frac{\partial v_{h}}{\partial y}\right|_{i_{p}, j_{p}}\right)\right. \\
& \left.+\frac{12}{c_{0} l}\left(\sum_{r=i_{p} \pm 1 / 2 ; s=j_{p} \pm 1 / 2} v_{r, s}^{2}\right)\right\} \text {, } \\
& v_{h}^{2}(M) l_{M} \leqslant \frac{2}{c_{0}^{2}}\left[\left(\sum_{p=0}^{N-1} v_{i_{p}+1 / 2, j_{p}+1 / 2}^{2} S_{i_{p}+1 / 2, j_{p}+1 / 2}\right)^{1 / 2}\right. \\
& +\left(\sum_{p=0}^{N-1} v_{i_{p}-1 / 2, j_{p}+1 / 2}^{2} S_{i_{p}-1 / 2, j_{p}+1 / 2}\right)^{1 / 2} \\
& +\left(\sum_{p=0}^{N-1} v_{i_{p}-1 / 2, j_{p}-1 / 2}^{2} S_{i_{p}-1 / 2, j_{p}-1 / 2}\right)^{1 / 2} \\
& \left.+\left(\sum_{p=0}^{N-1} v_{i_{p}+1 / 2, j_{p}-1 / 2}^{2} S_{i_{p}+1 / 2, j_{p}-1 / 2}\right)^{1 / 2}\right] \ldots \\
& \cdots \times\left[\left(\sum_{p=0}^{N-1}\left|\frac{\partial v_{h}}{\partial x}\right|_{i_{p}, j_{p}}^{2} S_{i_{p}, j_{p}}\right)^{1 / 2}+\left(\sum_{p=0}^{N-1}\left|\frac{\partial v_{h}}{\partial y}\right|_{i_{p}, j_{p}}^{2} S_{i_{p}, j_{p}}\right)^{1 / 2}\right] \\
& +\frac{48}{c_{0}^{2} l}\left[\sum_{p=0}^{N-1}\left(\sum_{r=i_{p} \pm 1 / 2 ; s=j_{p} \pm 1 / 2} v_{r, s}^{2} S_{r, s}\right)\right] \text {. }
\end{aligned}
$$

In order to derive an upper bound for $\left|v_{h}\right|_{\Gamma_{h}}^{2}$, the last inequality must be summed over all $M$ of $\dot{\Gamma}_{h}$. In doing so, one must evaluate the maximum number of paths, corresponding to the different nodes of $\dot{\Gamma}_{h}$, that can cross a given cell. Now, to each $M$, there corresponds a different path $P_{M}$ and a different parallelogram $T_{M}$ such that each cell crossed by $P_{M}$ cuts $T_{M}$. Hence, it suffices to evaluate the maximum number of parallelograms that can be cut by a given cell. The width of a cell is always smaller than $2 h$, and, according to (4.4) and (5.5), the width of a parallelogram $T_{M}$ is always greater than $\sigma_{0} h \sin \lambda$. Therefore, a cell can intersect at most $2 / \sigma_{0} \sin \lambda$ parallelograms in one direction. As there are $n$ directions, it follows that a cell can be crossed at most by $2 n / \sigma_{0} \sin \lambda$ different paths. This constant is independent of $h$. Therefore,

$$
\begin{aligned}
\left|v_{h}\right|_{\Gamma_{h}}^{2} \leqslant \frac{8 n}{c_{0}^{2} \sigma_{0} \sin \lambda}\left[2 \sum_{M \in \dot{O}_{h}} v_{h}^{2}(M) S_{M}+\sum_{K \in Q_{h}}\left\{\left(\frac{\partial v_{h}}{\partial x}\left(B_{K}\right)\right)^{2}\right.\right. & \left.+\left(\frac{\partial v_{h}}{\partial y}\left(B_{K}\right)\right)^{2}\right\} S_{K} \\
& \left.+\frac{48}{l} \sum_{M \in \dot{O}_{h}} v_{h}^{2}(M) S_{M}\right]
\end{aligned}
$$


The theorem is proved since all constants of the right-hand side are independent of $h$ and $v_{h}$.

Remark 5.3. From Theorem 5.2 and Lemma 3.5 we derive property (2.4) of $L_{h}$ :

$$
\left|L_{h}\left(v_{h}\right)\right| \leqslant \mu\left\|v_{h}\right\|_{h} \quad \forall v_{h} \in V_{h},
$$

where $\mu$ is a constant independent of $h$.

6. Error Estimate and Conclusion.

6.1. Order of the Approximation. The results of the preceding paragraphs are summed up in the next theorem which shows that our approximation is of order one.

THEOREM 6.1. Under the following hypotheses on the continuous problem (1.1) (1.2):

$$
\left\{\begin{array}{l}
\text { ellipticity of coefficients: } \mathrm{H} 1 \\
\left\|b_{i}\right\|_{L^{\infty}(\Omega)} \text { sufficiently small, }
\end{array}\right.
$$

$$
\left\{\begin{array}{l}
\text { regularity of coefficients: } \mathrm{H} 2, \\
\text { regularity of boundary: } \mathrm{H} 3, \\
\text { regularity of solution: } u \in \mathrm{C}^{3}(\bar{\Omega}),
\end{array}\right.
$$

and the following hypotheses on the quadrangulation $2_{h}$ :

$$
\text { good approximation of boundary: (3.3), }
$$

$$
\text { cartesian numbering of nodes: (3.4), }
$$

there exists a constant $C$, independent of $h$, such that, for all $h$ sufficiently small,

$$
\left\|u-u_{h}\right\|_{h} \leqslant C h,
$$

where $u_{h}$ denotes the solution of the approximate problem $(2.5)(3.8)(3.9)$ or (2.5) (3.8) $\left(3.9^{\prime}\right)$.

Proof. The hypotheses of Theorem 2.1 have been checked in Section 3; therefore, inequality (2.6) holds:

$$
\left\|u-u_{h}\right\|_{h} \leqslant C_{1}\left\{\inf _{v_{h} \in V_{h}}\left\|u-v_{h}\right\|_{h}+\sup _{w_{h} \in V_{h}} \frac{1}{\left\|w_{h}\right\|_{h}}\left|L_{h}\left(w_{h}\right)-a_{h}\left(u, w_{h}\right)\right|\right\},
$$

where $C_{1}$ is a constant independent of $h$.

An upper bound for the projection error has been given in Section 3: 


$$
\inf _{v_{h} \in V_{h}}\left\|u-v_{h}\right\|_{h} \leqslant C_{2} h,
$$

where $C_{2}$ is a constant independent of $h$.

Therefore, there remains to establish an upper bound for

$$
\frac{1}{\left\|w_{h}\right\|_{h}}\left|L_{h}\left(w_{h}\right)-a_{h}\left(u, w_{h}\right)\right|, \text { for any } w_{h} \neq 0 \text { in } V_{h} .
$$

Now,

$$
\begin{aligned}
a_{h}\left(u, w_{h}\right)= & {\left[a_{11} \frac{\partial u}{\partial x}+a_{12} \frac{\partial u}{\partial y}, \frac{\partial w_{h}}{\partial x}\right]_{h}+\left[a_{21} \frac{\partial u}{\partial x}+a_{22} \frac{\partial u}{\partial y}, \frac{\partial w_{h}}{\partial y}\right]_{h} } \\
& +\left[b_{1} \frac{\partial u}{\partial x}+b_{2} \frac{\partial u}{\partial y}, w_{h}\right]_{h}+\left(d u, w_{h}\right)_{h} .
\end{aligned}
$$

Combining Proposition 4.3 and Theorem 5.2, we get,

$$
\begin{aligned}
a_{h}\left(u, w_{h}\right)= & -\left(\frac{\partial}{\partial x}\left(a_{11} \frac{\partial u}{\partial x}+a_{12} \frac{\partial u}{\partial y}\right), w_{h}\right)_{h} \cdots \\
\cdots+ & {\left[\left(a_{11} \frac{\partial u}{\partial x}+a_{12} \frac{\partial u}{\partial y}\right) \cos (\vec{n}, x), w_{h}\right]_{\Gamma_{h}} } \\
& -\left(\frac{\partial}{\partial y}\left(a_{21} \frac{\partial u}{\partial x}+a_{22} \frac{\partial u}{\partial y}\right), w_{h}\right)_{h} \\
& +\left[\left(a_{21} \frac{\partial u}{\partial x}+a_{22} \frac{\partial u}{\partial y}\right) \cos (\vec{n}, y), w_{h}\right]_{\Gamma_{h}}+O(h)\left\|w_{h}\right\|_{h} \cdots \\
\cdots+ & {\left[b_{1} \frac{\partial u}{\partial x}+b_{2} \frac{\partial u}{\partial y}, w_{h}\right]_{h}+\left(d u, w_{h}\right)_{h}, \text { for sufficiently small } h }
\end{aligned}
$$

Let $L_{h}$ be defined by (3.9). Then

$$
L_{h}\left(w_{h}\right)=\left[f, w_{h}\right]_{h}+\left[g, w_{h}\right]_{\Gamma_{h}} .
$$

According to (1.1) and (1.2), this yields,

$$
\begin{aligned}
L_{h}\left(w_{h}\right)= & -\left[\frac{\partial}{\partial x}\left(a_{11} \frac{\partial u}{\partial x}+a_{12} \frac{\partial u}{\partial y}\right)+\frac{\partial}{\partial y}\left(a_{21} \frac{\partial u}{\partial x}+a_{22} \frac{\partial u}{\partial y}\right), w_{h}\right]_{h} \\
& +\left[b_{1} \frac{\partial u}{\partial x}+b_{2} \frac{\partial u}{\partial y}+d u, w_{h}\right]_{h} \\
& +\left[\left(a_{11} \frac{\partial u}{\partial x}+a_{12} \frac{\partial u}{\partial y}\right) \cos (\vec{n}, x), w_{h}\right]_{\Gamma_{h}} \\
& +\left[\left(a_{21} \frac{\partial u}{\partial x}+a_{22} \frac{\partial u}{\partial y}\right) \cos (\vec{n}, y), w_{h}\right]_{\Gamma_{h}} .
\end{aligned}
$$

Combining Lemma 4.4 and Theorem 5.2, we get, for $h$ small enough, 


$$
\begin{aligned}
L_{h}\left(w_{h}\right)=- & \left(\frac{\partial}{\partial x}\left(a_{11} \frac{\partial u}{\partial x}+a_{12} \frac{\partial u}{\partial y}\right)+\frac{\partial}{\partial y}\left(a_{21} \frac{\partial u}{\partial y}+a_{22} \frac{\partial u}{\partial y}\right), w_{h}\right)_{h} \\
+ & \left(d u, w_{h}\right)_{h}+O(h)\left\|w_{h}\right\|_{h}+\left[b_{1} \frac{\partial u}{\partial x}+b_{2} \frac{\partial u}{\partial y}, w_{h}\right]_{h} \cdots \\
& \cdots+\left[\left(a_{11} \frac{\partial u}{\partial x}+a_{12} \frac{\partial u}{\partial y}\right) \cos (\vec{n}, x), w_{h}\right]_{\Gamma_{h}} \\
+ & {\left[\left(a_{21} \frac{\partial u}{\partial x}+a_{22} \frac{\partial u}{\partial y}\right) \cos (\vec{n}, y), w_{h}\right]_{\Gamma_{h}} . }
\end{aligned}
$$

Therefore, for any function $w_{h}$ in $V_{h}$ and $h$ sufficiently small,

$$
L_{h}\left(w_{h}\right)=a_{h}\left(u, w_{h}\right)+O(h)\left\|w_{h}\right\|_{h},
$$

where the quantity $O(h)$ is independent of $w_{h}$.

Hence, if $L_{h}$ is defined by (3.9) and $h$ is sufficiently small,

$$
\sup _{w_{h} \in V_{h} ; w_{h} \neq 0} \frac{1}{\left\|w_{h}\right\|_{h}}\left|L_{h}\left(w_{h}\right)-a_{h}\left(u, w_{h}\right)\right| \leqslant C_{3} h,
$$

where $C_{3}$ is a constant independent of $h$.

Similarly, it can be shown that the same inequality holds, with a different constant, when $L_{h}$ is defined by $\left(3.9^{\prime}\right)$. Hence, the theorem is proved.

When the solution and coefficients do not satisfy the regularity hypotheses included in $n^{\circ} 2$, we cannot show the error estimate (6.1). However, if we replace these by:

$$
u \in H^{1}(\Omega), a_{i j}, b_{i}, d \text { and } f \in C^{0}(\bar{\Omega}) \text { and } g \in C^{0}(\Gamma),
$$

it can be shown, by a finite-difference approach, that

$$
\lim _{h \rightarrow 0}\left(u_{h}, \frac{\partial u_{h}}{\partial x}, \frac{\partial u_{h}}{\partial y},\left.u_{h}\right|_{\Gamma_{h}}\right)=\left(u, \frac{\partial u}{\partial x}, \frac{\partial u}{\partial y}, u_{\Gamma}\right)
$$

in appropriate discrete $L^{2}$ norms.

All the regularity hypotheses on $Q_{h}$ have already been encountered and discussed in our previous paper (cf. Girault [4]). Nevertheless, the following points are worth stressing. The two conditions, global regularity and mean regularity, do not occur in the classical finite element theory. They are an inevitable consequence of the nonellipticity of our approximation. In other words, failure to satisfy Strang's ellipticity condition must be compensated by additional regularity on the quadrangulation. It is interesting to note that these two hypotheses are satisfied when the cells of $2_{h}$ are nearly parallelograms, but this condition is not necessary.

6.2. Conclusion. From a practical point of view, the method we have studied in this paper is, by far, the simplest that can be derived in using quadrilateral finite elements: it leads to a nine-point scheme whose coefficients can be computed directly by a simple formula. Provided the quadrangulation is not too distorted, this method is of order one, a precision which is adequate in many applications. The important point of this paper has been to show that such a method could handle problems involving surface integrals without loss of accuracy. 
The study of nonelliptic approximation is now being extended to nonlinear problems.

Analyse Numérique, T55-65

Université Paris VI

75230 Paris, Cedex 05, France

1. J. H. BRAMBLE \& S. R. HILBERT, "Estimation of linear functionals on Sobolev spaces with application to Fourier transforms and spline interpolation," SIAM J. Numer. Anal., v. 7, 1970, pp. 112-124. MR 41 \#7819.

2. J. CEA, “Approximation variationnelle des problèmes aux limites," Ann. Inst. Fourier (Grenoble), v. 14, 1964, fasc. 2, pp. 345-444. MR 30 \#037.

3. P. G. CIARLET \& P. A. RAVIART, "The combined effect of curved boundaries and numerical integration in isoparametric finite element methods," The Mathematical Foundations of the Finite Element Method with Applications to Partial Differential Equations, A. K. Aziz (editor), Academic Press, New York, 1972, pp. 409-474.

4. V. GIRAULT, "Theory of a finite difference method on irregular networks," SIAM J. Numer. Anal., v. 11, 1974, pp. 260-282.

5. C. W. HIRT, A. A. AMSDEN \& J. L. COOK, "An arbitrary Lagrangian-Eulerian computing method for all flow speeds," J. Computational Phys., v. 14, 1974, pp. 227-253.

6. J. L. LIONS, "Problèmes aux limites en théorie des distributions," Acta Math., v. 94, 1955, pp. 13-153. MR 17, 745.

7. J.-L. LIONS, Problèmes aux Limites dans les Équations aux Dérivées Partielles, 2ième éd., Séminaire de Mathématiques Supérieures, no. 1 (Été, 1962), Presses Univ. Montréal, Montreal, Que., 1965. MR 40 \#4602.

8. J.-L. LIONS, Equations Diffèrentielles Opérationnelles et Problèmes aux Limites, Die Grundlehren der math. Wissenschaften, Band 111, Springer-Verlag, Berlin and New York, 1961. MR 27 \#3935.

9. G. STRANG, "Variational crimes in the finite element method," The Mathematical Foundations of the Finite Element Method with Applications to Partial Differential Equations, A. K. Aziz (editor), Academic Press, New York, 1972, pp. 689-710.

10. G. STRANG \& G. FIX, An Analysis of the Finite Element Method, Prentice-Hall, Englewood Cliffs, N. J., 1973. 